\title{
Design of Multilayer Dielectric Cover to Enhance Gain and Efficiency of Slot Arrays
}

\author{
Giorgio Montisci, ${ }^{1}$ Zusheng Jin, ${ }^{2}$ Mingchao Li, ${ }^{2}$ Hu Yang, \\ Giovanni Andrea Casula, ${ }^{1}$ Giuseppe Mazzarella, ${ }^{1}$ and Alessandro Fanti ${ }^{1}$ \\ ${ }^{1}$ Dipartimento di Ingegneria Elettrica ed Elettronica, Università di Cagliari, Piazza D’Armi, 09123 Cagliari, Italy \\ ${ }^{2}$ College of Electronics Science and Engineering, National University of Defense Technology, Changsha 410073, China \\ Correspondence should be addressed to Zusheng Jin; jin_hexi@126.com
}

Received 21 March 2013; Accepted 15 May 2013

Academic Editor: Massimiliano Pieraccini

Copyright (C) 2013 Giorgio Montisci et al. This is an open access article distributed under the Creative Commons Attribution License, which permits unrestricted use, distribution, and reproduction in any medium, provided the original work is properly cited.

\begin{abstract}
An effective design procedure, based on the Elliot synthesis method, is proposed to investigate the properties of waveguide slot arrays with multilayer dielectric cover. Then, the features of the designed arrays are analyzed by means of a FEM commercial software, namely, Ansys HFSS 13. We show how a proper choice of the dielectric cover configuration allows increasing the array gain and aperture efficiency, while taking advantage of the properties of the radome structure, in terms of insulation, protection, and pressurization of the radiating waveguides. Therefore, a significant outcome of the optimal choice of the multilayer dielectric cover is the reduction of the number of slots with respect to an array radiating into free space with the same gain and efficiency, with a consequent reduction of the production cost.
\end{abstract}

\section{Introduction}

Microwave antennas can be realized using either reflectors or arrays of different kinds of radiating elements [1]. The latter have typically a smaller bandwidth, but they are more compact, lighter, and particularly suitable for low profile applications [2].

A common solution for the realization of high-performance antennas in the whole microwave frequency range is the use of waveguide slot arrays [3-7]. This choice provides high gain, high efficiency, mechanical strength, and a very good polarization purity $[3,8]$. Many practical applications require that waveguide slot arrays are covered by a dielectric material either for protection or thermal insulation, aerodynamic purposes, or pressurization of the radiating waveguides [9].

The standard Elliott design procedure [3] for a waveguide array of longitudinal slots has been extended to a single-layer dielectric-covered slot array by Casula and Montisci [9] and Jin et al. [10]. However, sometimes a multilayer dielectric cover is required, as in the case of flat sandwich radomes used mainly for thermal insulation and protection [11].

Radomes are usually chosen and applied after the antenna has been designed and realized. Therefore, their geometric parameters, shape, and dielectric properties must be selected in order to reduce as much as possible their impact in the radiating and circuital properties of the antenna [12]. These precautions are not required if the radome is included in the antenna since the beginning, so that it can be taken into account in the analysis and design procedures. In this way, the performance of the designed antenna is not negatively affected by the presence of the radome structure. As a consequence, the geometry and dielectric properties of the radome can be selected for optimization of thermal insulation and protection or even designed in order to obtain the best tradeoff between the required antenna performance and the necessity of insulation or protection.

To address this task we need the following:

(a) a design procedure for an array of slots with a multilayer dielectric cover; 
(b) a criterion to choose the dielectric cover configuration in order to optimize the radiating properties of the antenna.

The choice of point (b) must be made among the set of covers able to give the required mechanical and thermal behavior. The determination of such set is, of course, outside the scope of this work.

Then, once a solution is available for the above points (a) and (b), the antenna designer is fully aware of all the implications of the choice of a particular configuration of radome (from both electromagnetic, thermodynamics, and material science point of view) and has enough information to achieve the optimal tradeoff based on the design specifications.

Therefore, first of all we need to extend the Elliott design procedure to the case of a multilayer dielectric-covered waveguide slot array, complying with (a). This task has been performed in a very effective manner, exploiting the wide experience of the authors' research groups on waveguide slot antennas $[5,6,9,10]$. Then, the array design procedure has been used to investigate the behavior of a multilayer dielectric-covered array.

The aim of this work is to show how a proper choice of the multilayer dielectric cover allows achieving the desirable insulation and protection, while improving the antenna gain and aperture efficiency. The basic idea of the proposed solution has been introduced by Von Trentini [13] in 1956. In that paper, the author studies the increase in directivity of an array of circular apertures by placing a partially reflecting sheet in front of the antenna, exploiting the multiple reflections between the antenna ground plane and the partially reflecting sheet. More recently, this strategy has been applied to microstrip antennas by many authors (see, e.g., [14-17]). However, to the best of the authors' knowledge, a discussion on the use of a multilayer dielectric cover to improve gain and efficiency of waveguide slot arrays is not available in the literature. This is quite surprising since the improvement for waveguide slot arrays is large because of their very high manufacturing cost compared, for example, to microstrip antennas. In this contest, a suitable configuration of the dielectric cover provides a reduction of the number of slots with respect to an array radiating into free space with the same gain and efficiency, thus allowing a significant reduction of the production cost. Actually, compared to an array radiating into free space, an optimized dielectric covered array results in a low increase of the manufacturing cost for the realization of the dielectric cover against a significant saving due to the reduction of the number of slots and radiating guides and the simplification of the feeding network.

In this paper, we have considered a slot array with a single feeding waveguide $[9,18]$. However, the results of this work apply also to arrays with different feeding networks, wherein the configuration used in this paper can be considered as a subarray of a more complex structure [19-21].

The properties of the designed dielectric-covered arrays have been investigated by using Ansys HFSS, which, at least for waveguide slot arrays, can be considered equivalent to experiment as reported, for example, in [19, 22].

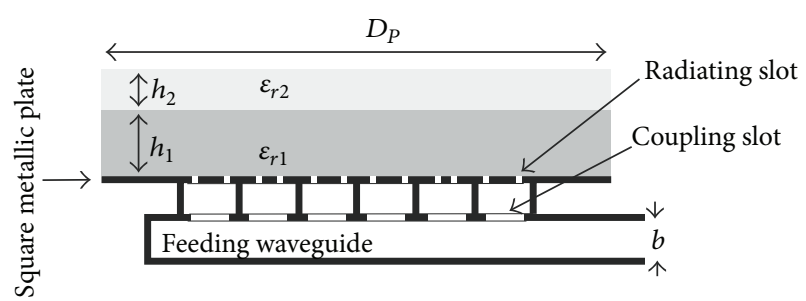

(a)

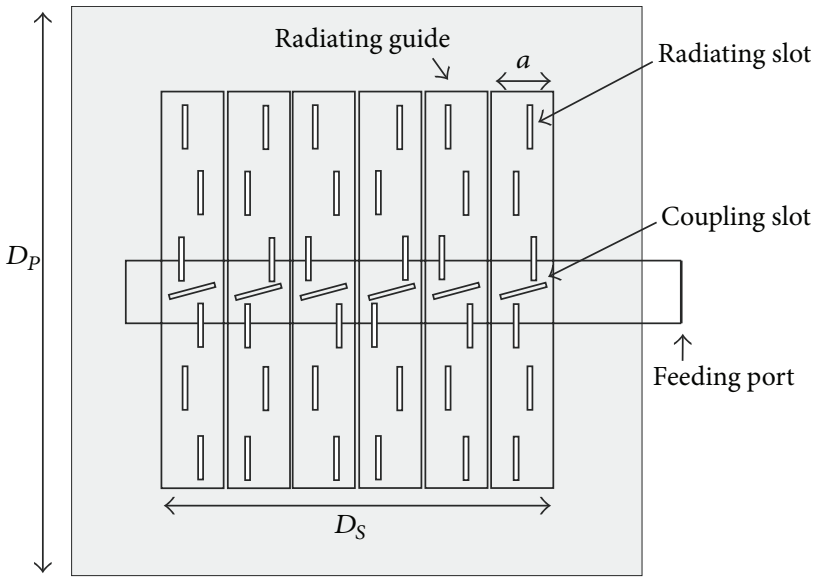

(b)

Figure 1: Array geometry: (a) side view; (b) top view.

\section{Design of the Dielectric Cover}

The reasons for a dielectric cover are usually insulation and protection so that it is considered as a parasitic structure and, therefore, the smaller its effect on the antenna behavior, the best the cover. However, since in many cases its presence is needed, we can face the problem the other way, that is, by using the dielectric cover to improve the antenna performance, and, in particular, as we will show next in this section, maximize the array gain. In order to illustrate this feature, we select here the basic configuration of a two-layer dielectric cover (see Figure 1(a)), designed according to [13].

The first layer is a substrate with low dielectric permittivity (air or foam) of proper thickness, and the second layer is a substrate with high-dielectric permittivity acting as a partially reflecting sheet. Let the reflection coefficient of this sheet in the broadside direction be $p \exp (j \psi)$.

We define by $h_{1}, \varepsilon_{r 1}$ and $h_{2}, \varepsilon_{r 2}$, the thicknesses and dielectric permittivities of the first and second layer, respectively (Figure 1). The thickness of the first layer has been chosen in order to ensure the maximum radiated power in the broadside direction. As derived in [13], the radiated power density is

$$
S(\vartheta)=\frac{1-p^{2}}{1+p^{2}-2 p \cos \left(\psi-\pi-\left(4 \pi h_{1} / \lambda\right) \cos \vartheta\right)},
$$

wherein $\vartheta$ is the angle from broadside and $\lambda=\lambda_{0} / \sqrt{\varepsilon_{r 1}}, \lambda_{0}$ being the free-space wavelength. Of course, in (1) the amplitude $p$ and the phase $\psi$ of the reflection coefficient of the sheet 
are functions of the angle $\vartheta$. For a pencil-beam slot array, we are interested in the case $\vartheta=0^{\circ}$ (broadside direction).

The maximum $S(0)$ can be obtained by maximizing (1), which gives

$$
\psi-\pi-\frac{4 \pi h_{1}}{\lambda}=2 N \pi
$$

wherein $N$ is integer.

Equation (2) allows computing the optimal dielectric thickness as

$$
h_{1}=\left(\frac{\psi}{2 \pi}-0.5+N\right) \frac{\lambda_{0}}{2 \sqrt{\varepsilon_{r 1}}},
$$

wherein $N$ is chosen to obtain the smallest positive $h_{1}$.

The maximum power depends on $p^{2}$, too. As a matter of fact, the second layer acts as a partially reflecting sheet and, as shown in [13], the larger the reflectivity $p^{2}$ of this sheet, the higher the array gain. This is achieved when $\varepsilon_{r 2}$ is as high as possible (in the following we choose it equal to 9.8) and $h_{2}$ is $\lambda_{0} /\left(4 \sqrt{\varepsilon_{r 2}}\right)$ as reported also in [16] for the case of a microstrip antenna.

The presence of the dielectric cover modifies the radiating properties and the input matching of the array. This effect can be minimized using accurate design procedures, based on the Elliott model, and including the radome in the antenna structure [9] allows achieving the required input matching and far-field pattern. To improve the antenna behavior, a closer look to the performance of a double-layer dielectric cover for a slot array is in order. More precisely, in the next section we will show how a cover, designed according to (1)(3), can improve the array aperture efficiency (and gain).

\section{Performance of the Dielectric-Covered Arrays}

We tested the proposed solution on the following examples:

(a) $6 \times 6$ planar array with two-layer dielectric cover;

(b) $8 \times 8$ planar array radiating into free space;

(c) $8 \times 8$ planar array with two-layer dielectric cover;

(d) $10 \times 10$ planar array radiating into free space.

Since the reduction in the number of slots is a significant outcome of our discussion, we will compare not only arrays (b) and (c) but also array (a) with (b) and array (c) with (d).

As a matter of fact, a free-space slot array and a dielectriccovered one do not compete for the same applications. So, an array with the two-layer cover considered here should be compared with a standard dielectric-covered array [9]. However, the performance of a free-space array is better than or equal to the one of a single-layer dielectric-covered array with the same size and number of slots. Therefore, we decided to use the former in the comparison, for sake of clarity.

The design of the dielectric-covered arrays has been done by using the standard Elliott iterative technique extended to the case of a multilayer dielectric cover. The procedure described in [23] has been used for the computation of the slot self-admittance, but evaluating the Green's function in the external region as reported in [24]. The external mutual coupling has been computed with the same approach presented in [10], extended to the multilayer case.

The operating frequency has been selected equal to $9 \mathrm{GHz}$ and, for all the examples considered here, we have required side lobes below $-20 \mathrm{~dB}$. However, in the dielectric cover cases the excitations of the slots must be chosen in order to take into account that each slot radiates into a half space with a thick dielectric cover and the effect of the element factor cannot be neglected. In fact, the principle of "multiple reflections" applies to the field of the single radiating slot as well and strongly affects the array pattern. Therefore, in order to achieve the required amplitude distribution, the element factor has been properly taken into account by using a procedure for array pattern synthesis with the constraint of real excitations [25].

The chosen array geometry is shown in Figure 1, and employs a popular feeding technique [9]. Both the radiating and the feeding waveguides are half-height WR90 $(a=$ $22.86 \mathrm{~mm}, b=5.08 \mathrm{~mm}$, and waveguide wall thickness = $1 \mathrm{~mm}$ ), the length of the coupling slots is $17.1 \mathrm{~mm}$, their width is $1.5 \mathrm{~mm}$, and the tilt angle is $15^{\circ}$. The width of the radiating slots is $1.58 \mathrm{~mm}$.

Let us define $D_{S}$ as the side of the smallest square region that includes all the radiating guides and $D_{P}$ as the side of the square metallic plate in which the slots are cut. The dielectric cover lies on this metallic plate and has, therefore, the same square extension with side $D_{P}$ (see Figure 1(a)). Of course $D_{P} \geq D_{S}$, and the latter is dictated by the guide width and by the number of slots. For the arrays radiating into free space, $D_{P}$ has been chosen about $15 \mathrm{~mm}$ larger than $D_{S}$ in order to avoid second order effects in the farfield pattern due to the finite extension of the metallic plate. On the other hand, for the arrays with the dielectric cover, $D_{P}$ is a degree of freedom and must be suitably chosen in order to fully exploit the principle of multiple reflections, and then maximize the array gain $G$ and its aperture efficiency, defined as $\eta_{A}=G \lambda^{2} /\left(4 \pi D_{P}^{2}\right)$, wherein $\lambda$ is the free-space wavelength.

According to the design rules of Section 2, the following parameters have been selected for the two-layer dielectric cover: (i) first layer (foam): $\varepsilon_{r 1}=1.093, \tan \delta=0.0038, h_{1}=$ $15.8 \mathrm{~mm}$; (ii) second layer: $\varepsilon_{r 2}=9.8, \tan \delta=0.002, h_{2}=$ $2.54 \mathrm{~mm}$.

The designed arrays have been simulated with Ansys HFSS. The HFSS model takes into account the finite size of the ground plane, in which the radiating slots are cut, by using a radiation box which encloses the entire structure depicted in Figure 1. The dielectric losses have also been included in this model.

The main results are shown in Figures 2 and 3, where the gain and efficiency of an array with the two-layer dielectric cover are evaluated for different $D_{P}$. It appears that the highest gain is obtained for $D_{P}$ equal to $210 \mathrm{~mm}$ for a $6 \times 6$ array and equal to $260 \mathrm{~mm}$ for an $8 \times 8$ array. The corresponding values are shown in Table 1, together with the values for arrays 
TABLE 1: Array gain and aperture efficiency.

\begin{tabular}{lccccc}
\hline Array type & $\begin{array}{c}\text { Dielectric } \\
\text { cover }\end{array}$ & $\begin{array}{c}D_{S} \\
(\mathrm{~mm})\end{array}$ & $\begin{array}{c}D_{P} \\
(\mathrm{~mm})\end{array}$ & $\begin{array}{c}\text { Gain } \\
(\mathrm{dB})\end{array}$ & $\eta_{A}$ \\
\hline (a) $6 \times 6$ & 2-layer & 148.43 & 170 & $24.2 \mathrm{~dB}$ & $80 \%$ \\
(a) $6 \times 6$ & 2-layer & 148.43 & 190 & $24.6 \mathrm{~dB}$ & $70 \%$ \\
(a) $6 \times 6$ & 2-layer & 148.43 & 210 & $25 \mathrm{~dB}$ & $64 \%$ \\
\hline (b) $8 \times 8$ & NO & 197.04 & 210 & $25.1 \mathrm{~dB}$ & $65 \%$ \\
\hline (c) $8 \times 8$ & 2-layer & 197.04 & 210 & $26.4 \mathrm{~dB}$ & $87 \%$ \\
(c) $8 \times 8$ & 2-layer & 197.04 & 240 & $26.8 \mathrm{~dB}$ & $73 \%$ \\
(c) $8 \times 8$ & 2-layer & 197.04 & 260 & $27.1 \mathrm{~dB}$ & $67 \%$ \\
\hline (d) $10 \times 10$ & NO & 245.69 & 260 & $27.2 \mathrm{~dB}$ & $69 \%$ \\
\hline
\end{tabular}

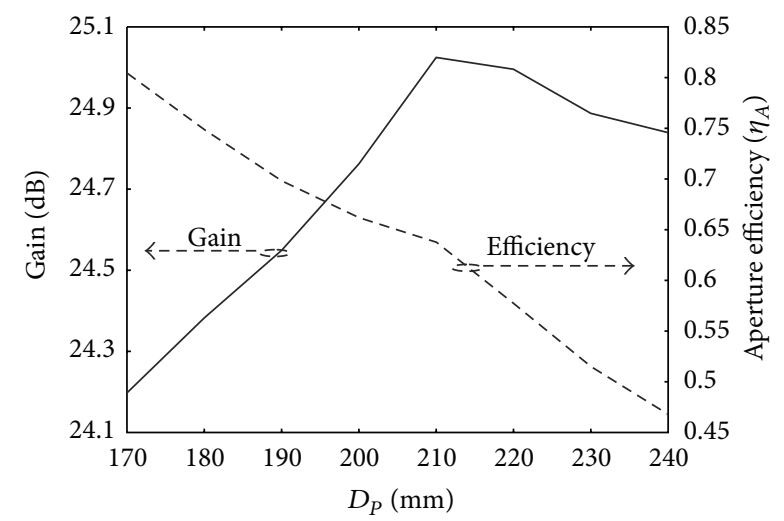

FIGURE 2: $6 \times 6$ array with two-layer dielectric cover: gain and efficiency as a function of $D_{P}$.

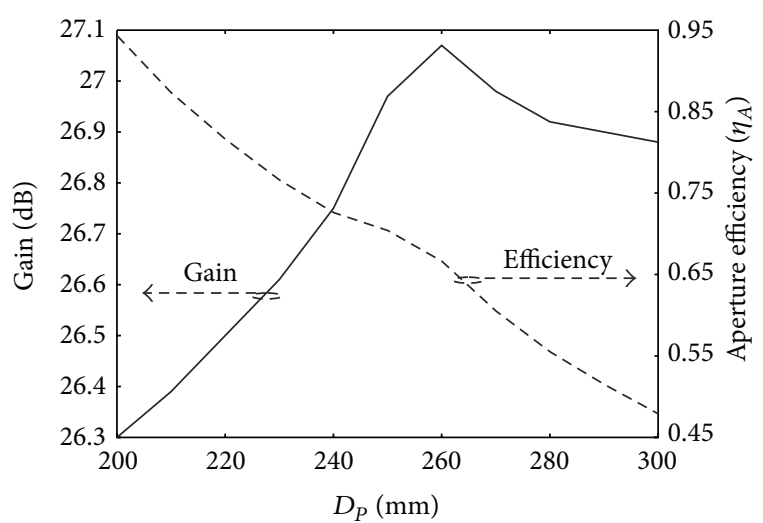

FIGURE 3: $8 \times 8$ array with two-layer dielectric cover: gain and efficiency as a function of $D_{P}$.

without the dielectric cover. The results for some arrays with a smaller $D_{P}$ are also shown, of course only for the dielectriccover case. It is clear that the gain depends essentially on the size $D_{P}$ of the ground plane, but the dielectric cover case achieves the same gain with a smaller number of slots and therefore allows for a smaller $D_{P}$. The importance of this consideration comes out looking at the efficiency curves of Figures 2 and 3 . It is clear that a reduction of $D_{P}$ causes a significant increase of the efficiency. In other words, the strongly reflecting dielectric cover allows a significantly better

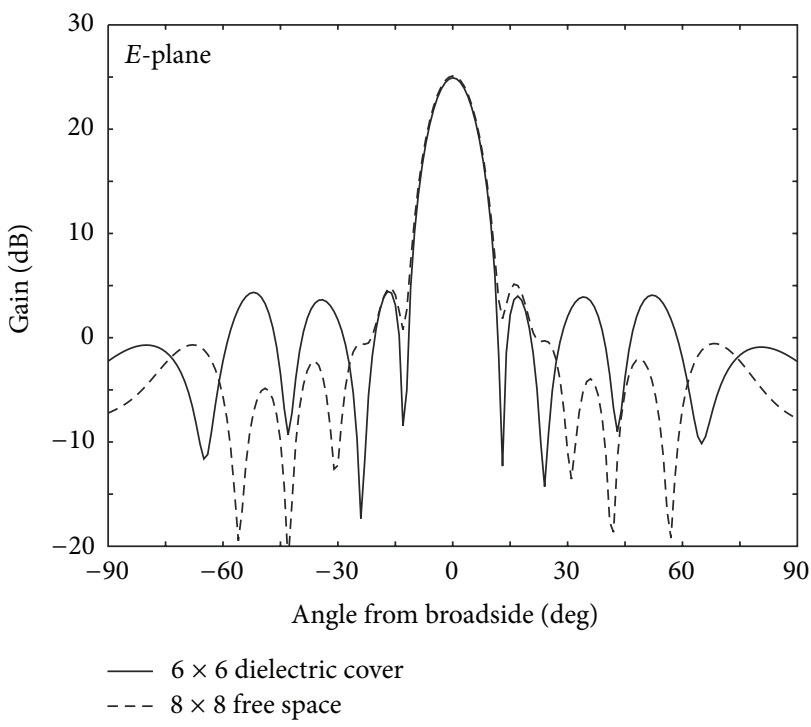

(a)

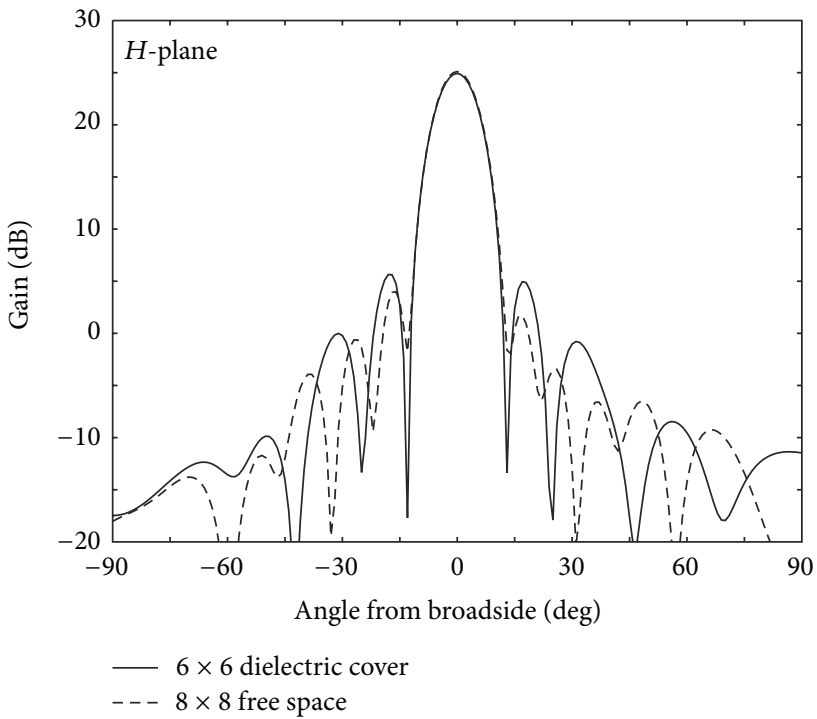

(b)

FIGURE 4: Simulated gain (HFSS) of $6 \times 6$ array with dielectric cover and $8 \times 8$ radiating in free space. $D_{P}=210 \mathrm{~mm}$.

use of the ground plane extension. The comparison of the data in Table 1 shows that the efficiency of an $8 \times 8$ array with a ground plane of $210 \mathrm{~mm}$ increases from $65 \%$, for the free-space array, to $87 \%$, for the array with dielectric cover, whereas the gain increases of about $1.3 \mathrm{~dB}$. Of course, this is true as long as the presence of the dielectric cover is taken into account from the beginning into the design procedure [9].

In order to complete the evaluation, in Figure 4 we show the comparison between the far-fields of arrays (a) and (b) for $D_{P}=210 \mathrm{~mm}$ and in Figure 5 the one between far-fields of arrays (c) and (d) for $D_{P}=260 \mathrm{~mm}$. Then, in Figure 6, the comparison of the frequency responses is also reported.

From the results of Table 1 and Figures 4, 5, and 6, it is apparent that an $8 \times 8$ array radiating into free space has the 


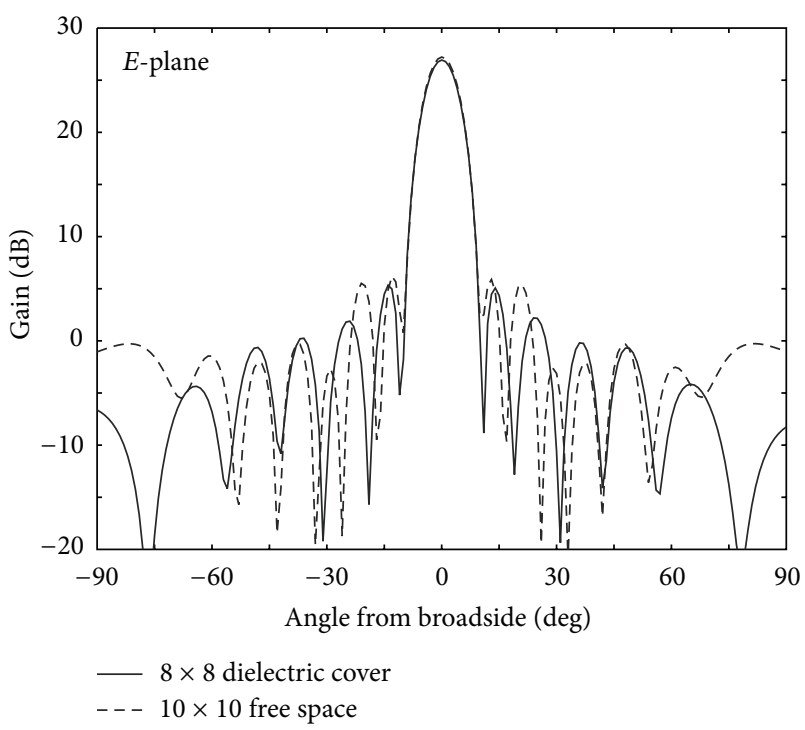

(a)

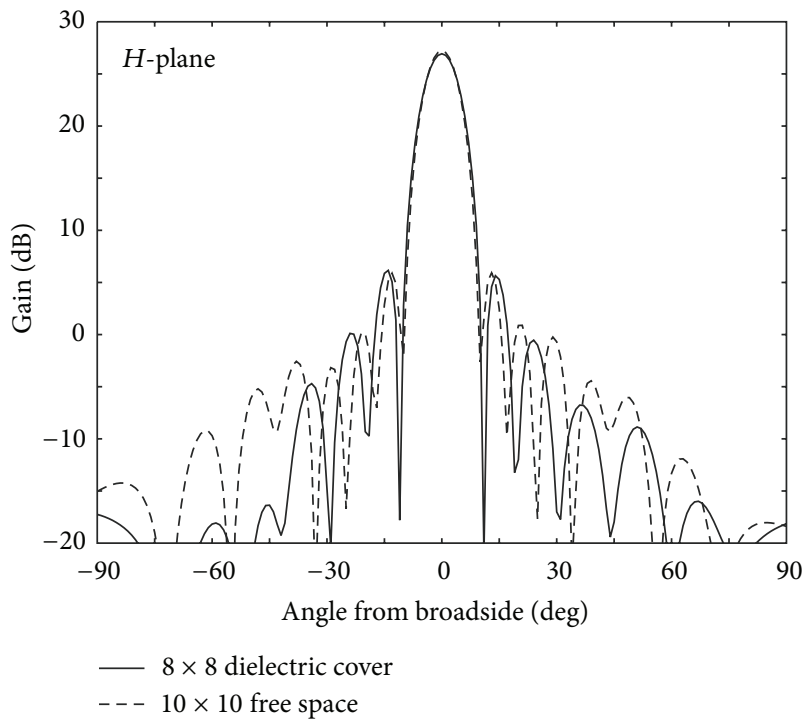

(b)

FIGURE 5: Simulated gain (HFSS) of $8 \times 8$ array with dielectric cover and $10 \times 10$ radiating in free space. $D_{P}=260 \mathrm{~mm}$.

same radiating properties and bandwidth of a $6 \times 6$ array with dielectric cover (with the same $D_{P}=210 \mathrm{~mm}$ ), and a $10 \times 10$ array radiating into free space is equivalent to an $8 \times 8$ array with dielectric cover (with the same $D_{P}=260 \mathrm{~mm}$ ).

Till now, we have considered only the case of a doublelayer dielectric cover. However, the same considerations can be carried out also for different dielectric cover configurations. For example, we can use a (three-layer) sandwich radome, wherein a further dielectric layer is inserted beneath the foam of the two-layer configuration, to increase the pressurization of the radiating waveguides [9]. In particular, if we add a layer with $\varepsilon_{r}=2.2, \tan \delta=0.0009$, and $h=0.381 \mathrm{~mm}$, we find that the thickness of the foam must

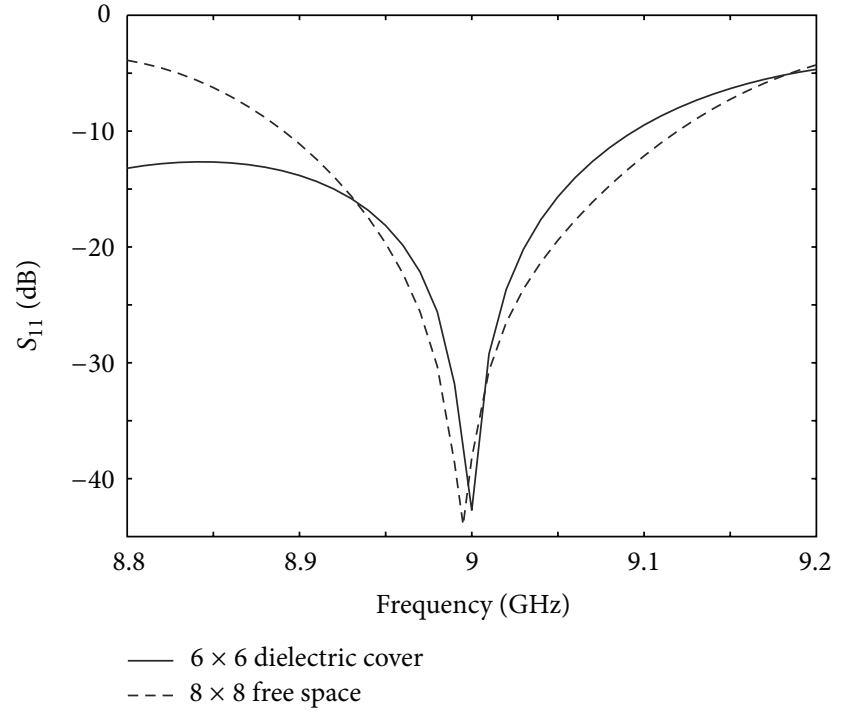

(a)

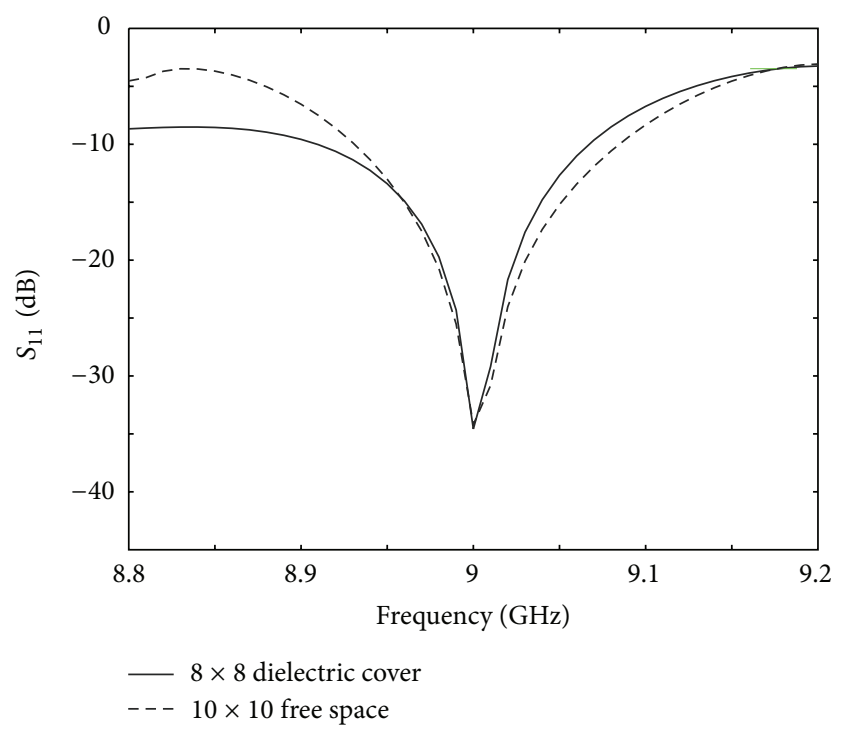

(b)

FIGURE 6: Frequency response (HFSS) of the designed arrays.

be slightly reduced to optimize the array gain (from $15.8 \mathrm{~mm}$ of the two-layer case to $15.6 \mathrm{~mm}$ ).

Both for a $6 \times 6$ array and for an $8 \times 8$ one, using this dielectric cover, we have found virtually the same optimal values of $D_{P}$ and array gain, and the same efficiency as in the case of the two-layer dielectric cover reported in Table 1.

\section{Conclusion}

In this paper, we show how to turn a potentially harmful structural requirement, the need for a dielectric radome, into an opportunity for planar slot arrays. The presented results show that a suitably designed dielectric cover allows reducing the number of slots by about one-third or even more, without 
affecting the array performance. This reduction and the consequent reduction in radiating guides and feeding networks will lower significantly the weight and the production cost, of a planar slot array.

\section{References}

[1] R. S. Elliot, Antenna Theory and Design, Prentice-Hall, New York, NY, USA, 1981.

[2] Y. T. Lo and S. W. Lee, Antenna Handbook Vol 3: Antenna Applications, Chapman \& Hall, New York, NY, USA, 1993.

[3] R. S. Elliott, "An improved design procedure for small arrays of shunt slots," IEEE Transactions on Antennas and Propagation, vol. 31, no. 1, pp. 48-53, 1983.

[4] Y. Kimura, T. Hirano, J. Hirokawa, and M. Ando, "Alternatingphase fed single-layer slotted waveguide arrays with chokes dispensing with narrow wall contacts," IEE Proceedings, vol. 148, no. 5, pp. 295-301, 2001.

[5] G. Montisci, "Design of circularly polarized waveguide slot linear arrays," IEEE Transactions on Antennas and Propagation, vol. 54, no. 10, pp. 3025-3029, 2006.

[6] S. Costanzo, G. A. Casula, A. Borgia et al., "Synthesis of slot arrays on integrated waveguides," IEEE Antennas and Wireless Propagation Letters, vol. 9, pp. 962-965, 2010.

[7] Y. Miura, J. Hirokawa, M. Ando, Y. Shibuya, and G. Yoshida, "Double-layer full-corporate-feed hollow-waveguide slot array antenna in the 60-GHz band," IEEE Transactions on Antennas and Propagation, vol. 59, pp. 2844-2851, 2011.

[8] G. Montisci, M. Musa, and G. Mazzarella, "Waveguide slot antennas for circularly polarized radiated field," IEEE Transactions on Antennas and Propagation, vol. 52, no. 2, pp. 619-623, 2004.

[9] G. A. Casula and G. Montisci, "Design of dielectric-covered planar arrays of longitudinal slots," IEEE Antennas and Wireless Propagation Letters, vol. 8, pp. 752-755, 2009.

[10] Z. Jin, G. Montisci, G. A. Casula, H. Yang, and J. Lu, "Efficient evaluation of the external mutual coupling in dielectric-covered waveguide slot arrays," International Journal of Antennas and Propagation, vol. 2012, Article ID 491242, 7 pages, 2012.

[11] A. Kedar and U. K. Revankar, "Parametric study of flat sandwich multilayer radome," Progress in Electromagnetics Research, vol. 66, pp. 253-265, 2006.

[12] R. Orta, R. Tascone, and R. Zich, "Performance degradation of dielectric radome covered antennas," IEEE Transactions on Antennas and Propagation, vol. 36, no. 12, pp. 1707-1713, 1988.

[13] G. Von Trentini, "Partially reflecting sheet arrays," IRE Transactions on Antennas and Propagation, vol. 4, pp. 666-671, 1956.

[14] N. G. Alexopoulos and D. R. Jackson, "Fundamental superstrate (cover) effects on printed circuit antennas," IEEE Transactions on Antennas and Propagation, vol. 32, no. 8, pp. 807-816, 1984.

[15] D. R. Jackson and N. G. Alexopoulos, "Gain enhancement methods for printed circuit antennas," IEEE Transactions on Antennas and Propagation, vol. AP-33, no. 9, pp. 976-987, 1985.

[16] F. Kaymaram and L. Shafai, "Enhancement of microstrip antenna directivity using double-superstrate configurations," Canadian Journal of Electrical and Computer Engineering, vol. 32, no. 2, pp. 77-82, 2007.

[17] R. Gardelli, M. Albani, and F. Capolino, "Array thinning by using antennas in a Fabry-Perot cavity for gain enhancement," IEEE Transactions on Antennas and Propagation, vol. 54, no. 7, pp. 1979-1990, 2006.
[18] G. Mazzarella and G. Montisci, "Accurate modeling of coupling junctions in dielectric covered waveguide slot arrays," Progress In Electromagnetics Research M, vol. 17, pp. 59-71, 2011.

[19] S. R. Rengarajan, M. S. Zawadzki, and R. E. Hodges, "Design, analysis, and development of a large Ka-band slot array for digital beam-forming application," IEEE Transactions on Antennas and Propagation, vol. 57, no. 10, pp. 3103-3109, 2009.

[20] G. A. Casula, G. Mazzarella, and G. Montisci, "Effect of the feeding T-junctions in the performance of planar waveguide slot arrays," IEEE Antennas and Wireless Propagation Letters, vol. 11, pp. 953-956, 2012.

[21] G. Mazzarella and G. Montisci, "Accurate characterization of the interaction between coupling slots and waveguide bends in waveguide slot arrays," IEEE Transactions on Microwave Theory and Techniques, vol. 48, no. 7, pp. 1154-1157, 2000.

[22] S. R. Rengarajan, M. S. Zawadzki, and R. E. Hodges, "Waveguide-slot array antenna designs for low-average-sidelobe specifications," IEEE Antennas and Propagation Magazine, vol. 52, no. 6, pp. 89-98, 2010.

[23] G. Mazzarella and G. Montisci, "Rigorous analysis of dielectriccovered narrow longitudinal shunt slots with finite wall thickness," Electromagnetics, vol. 19, no. 5, pp. 407-418, 1999.

[24] K. A. Michalski and J. R. Mosig, "Multilayered media green's functions in integral equation formulations," IEEE Transactions on Antennas and Propagation, vol. 45, no. 3, pp. 508-519, 1997.

[25] G. Franceschetti, G. Mazzarella, and G. Panariello, "Array synthesis with excitation constraints," IEE Proceedings $H$, vol. 135, no. 6 , pp. 400-407, 1988. 

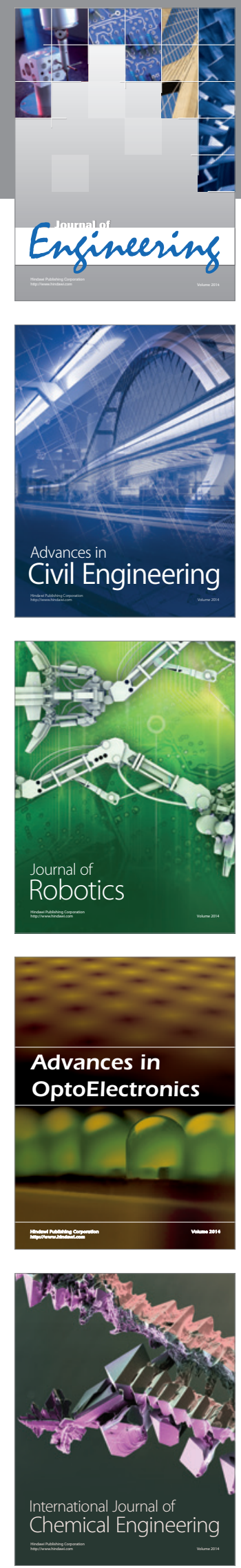

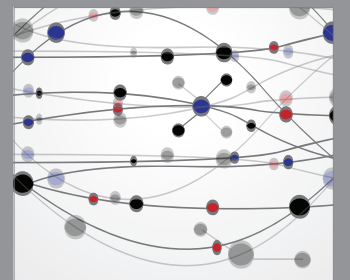

The Scientific World Journal
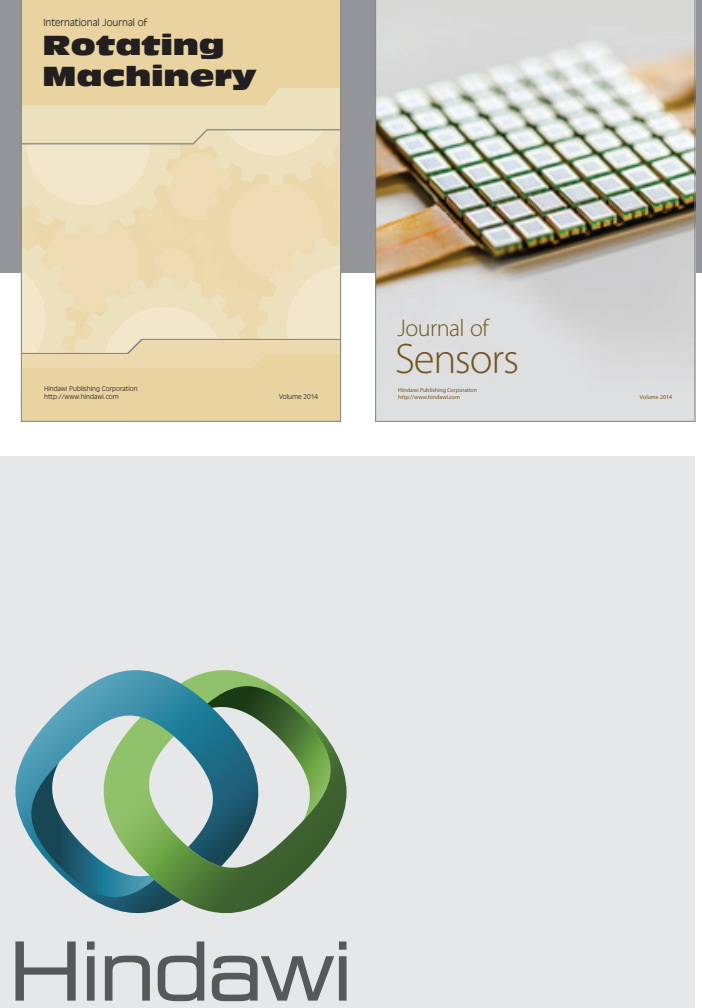

Submit your manuscripts at http://www.hindawi.com
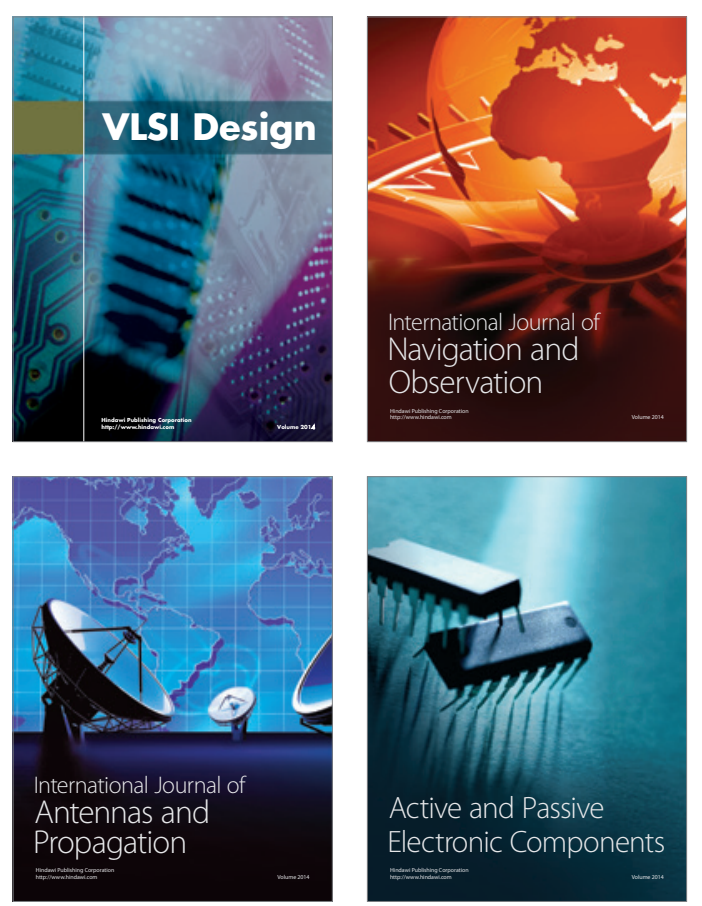
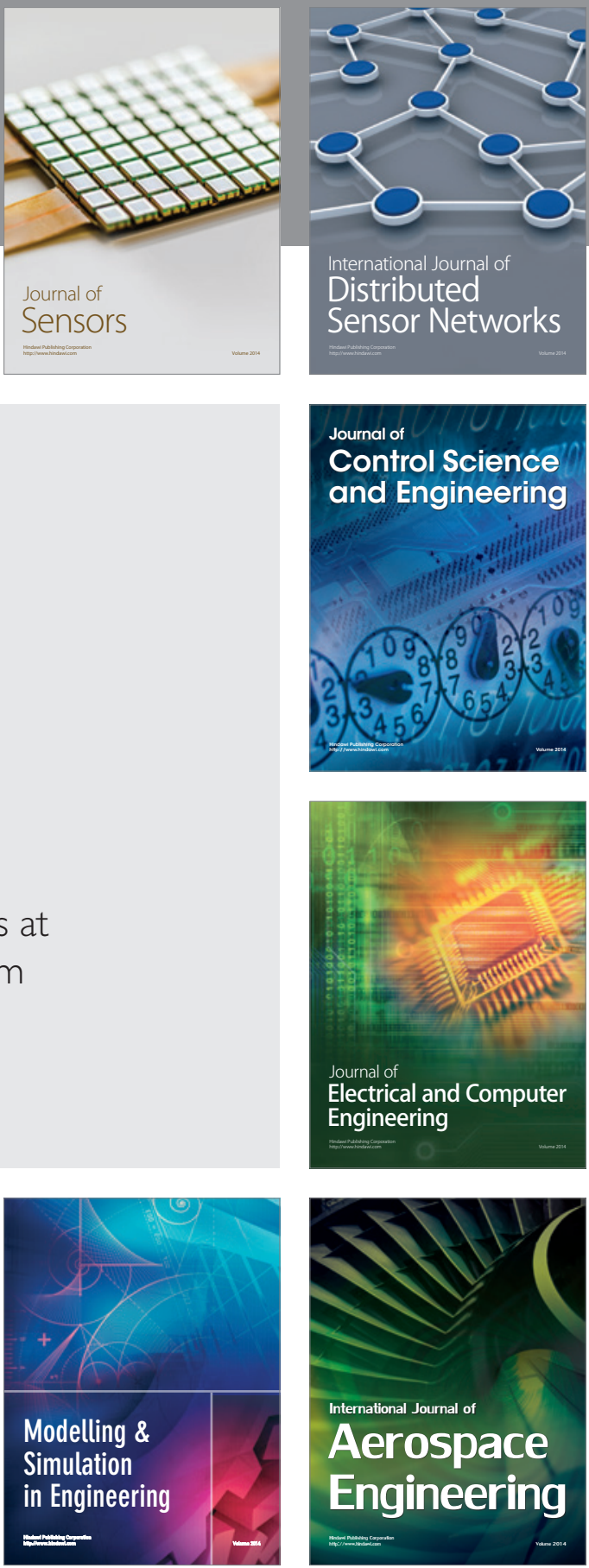

Journal of

Control Science

and Engineering
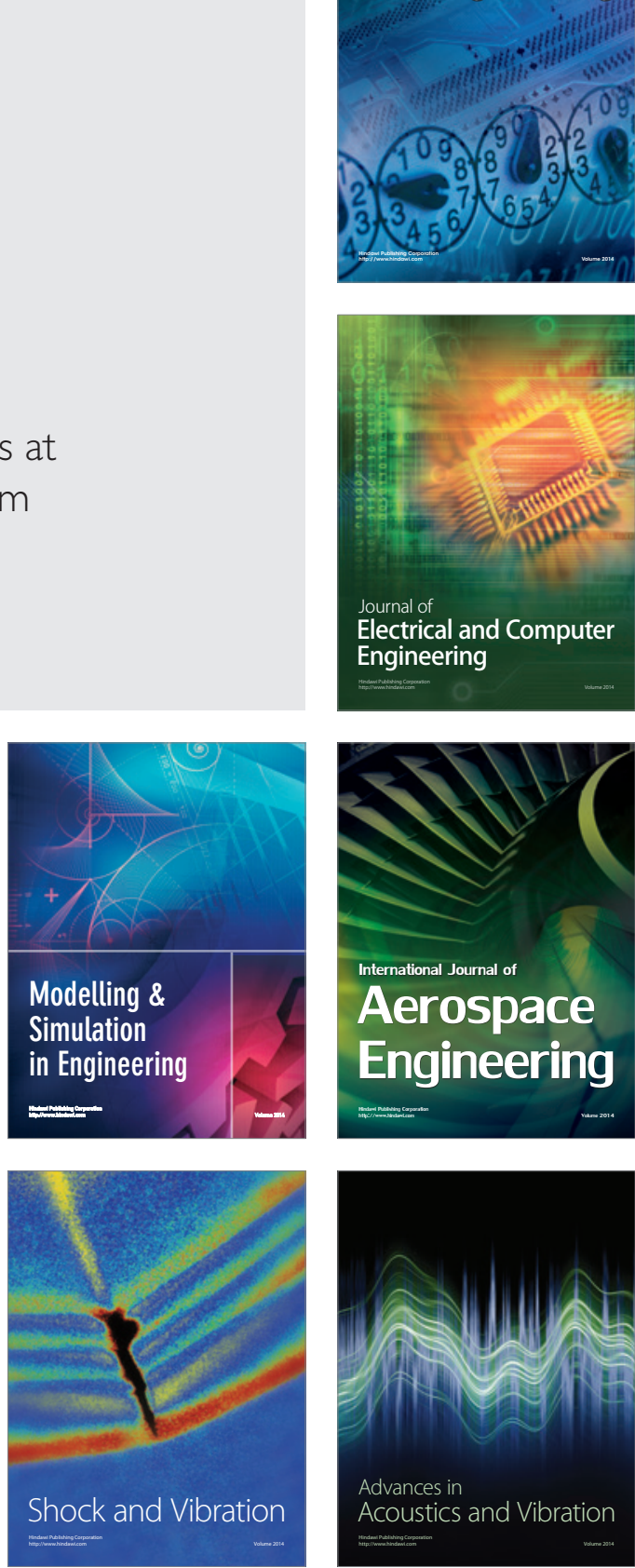